\title{
Unicity of meromorphic functions and their linear differential polynomials
}

\section{Chenguang Wang ${ }^{1 *}$ and Qi Han²}

"Correspondence:
cwang@jnxy.edu.cn
'Department of Mathematics,
Jining University, Qufu, Shandong
273155, P.R. China
Full list of author information is
available at the end of the article

\begin{abstract}
This paper studies the unicity of meromorphic functions that share an arbitrary nonzero small function with their general linear differential polynomials. The result derived here extends one by Brück in 1996 and others.

MSC: 30D35; 30D30

Keywords: Nevanlinna theory; meromorphic functions; small functions; entire functions; differential polynomials
\end{abstract}

\section{Introduction}

In 1929, R Nevanlinna [1-3] proved his celebrated five-value and four-value theorems, which gave birth to the study of the unicity of meromorphic functions in the open complex plane $\mathbb{C}$; in 1977 , by considering the unicity of an entire function $f$ and its first order derivative $f^{\prime}$, Rubel and Yang [4] showed that $f \equiv f^{\prime}$ when they share two distinct, finite values $C M$, which was generalized to meromorphic functions independently by Gundersen [5] as well as by Mues and Steinmetz [6] in 1983. Since these results, there have been many papers on several related problems about $f$ and its derivatives, and we refer the reader to $[3,7-13]$ and the references therein for more details.

Before we proceed, we spare the reader for a moment and assume the familiarity with the basics of Nevanlinna's value distribution theory of meromorphic functions in $\mathbb{C}$ such as the first and second main theorems, and the common notations such as the characteristic function $T(r, f)$, the proximity function $m(r, f)$ and the counting functions $N(r, f)$ (with multiplicities) and $\bar{N}(r, f)$ (without multiplicities); $S(r, f)$ denotes any quantity satisfying $S(r, f)=o(T(r, f))$ as $r \rightarrow \infty$ except possibly on a set of finite Lebesgue measure, not necessarily the same at each occurrence.

Let $a, f, g$ be some meromorphic functions on $\mathbb{C}$. $a$ is said to be a small function to $f$, provided $T(r, a)=S(r, f)$. Given $a$, a small function to both $f$ and $g$ or some value in $\mathbb{C} \cup$ $\{\infty\}$, one says that $f$ and $g$ share $a C M$ provided the two meromorphic functions $f-a$ and $g-a$ have the same zeros with counting multiplicities.

Now, we continue our discussion and observe a question that arises naturally: When only $f$ and $f^{\prime}$ share one value $C M$, then what can one have accordingly? Towards this direction, in 1996, Brück [14] first proved the following result.

๑ 2014 Wang and Han; licensee Springer. This is an Open Access article distributed under the terms of the Creative Commons Attribution License (http://creativecommons.org/licenses/by/2.0), which permits unrestricted use, distribution, and reproduction in any medium, provided the original work is properly cited. 
Theorem A Let $f$ be a nonconstant entire function with $N\left(r, \frac{1}{f^{\prime}}\right)=S(r, f)$. When $f$ and $f^{\prime}$ share a finite, nonzero value a CM, then there is a constant $c \neq 0$ such that

$$
\frac{f-a}{f^{\prime}-a}=c
$$

This interesting result was extended by Zhang [15] in 1998, when he proved, among some related results, the following one.

Theorem B Letf be a nonconstant meromorphic function such that

$$
\bar{N}(r, f)+N\left(r, \frac{1}{f^{\prime}}\right)<(\lambda+o(1)) T\left(r, f^{\prime}\right) \quad \text { for } \lambda \in\left(0, \frac{1}{2}\right) .
$$

When $f$ and $f^{\prime}$ share a finite, nonzero value a CM, then we have (1).

Quite recently, via replacing the finite, nonzero value $a$ by a small function $a(z)$ related to $f$, Al-Khaladi $[16,17]$ obtained the following striking result.

Theorem C Let $f$ be a nonconstant meromorphic function such that

$$
\bar{N}(r, f)+\bar{N}\left(r, \frac{1}{f^{\prime}}\right)<(\lambda+o(1)) T\left(r, f^{\prime}\right) \quad \text { for } \lambda \in\left(0, \frac{1}{2}\right) .
$$

When $f$ and $f^{\prime}$ share a small function $a(z)(\not \equiv 0, \infty) C M$, then we have $\frac{f-a}{f^{\prime}-a}=1-\frac{c}{a}$, where $c$ is a constant such that $\frac{c}{a} \neq 1$ and $N\left(r, 1-\frac{c}{a}\right)=S(r, f)$.

Remark D There are reasons why the condition on the zeros of $f^{\prime}$ is posed by Brück [14]: To control the multiple values of $f$ for each finite value, inspired by the exponential function; yet, it is not natural to generalize this condition by the zeros of $f^{(k)}$ when $k \geq 2$ as there is no need to control the multiple values of $f^{\prime}$.

Next, we define a linear differential polynomial of order $k \geq 1$, such as

$$
\mathcal{L}(f):=a_{1} f^{\prime}+a_{2} f^{\prime \prime}+\cdots+a_{k} f^{(k)} \quad\left(a_{k} \neq 0\right)
$$

associated with $f$, where $a_{j}$ are small functions of $f$ for $j=1,2, \ldots, k$. Then we can prove the following theorems, which are the main results of this paper.

Theorem 1 Let $f$ be a nonconstant meromorphic function satisfying (3) with $\lambda \in\left(0, \frac{1}{k+1}\right)$. When $f$ and $\mathcal{L}(f)$ share a small function $a(z)(\not \equiv 0, \infty) C M$, then we have $\frac{f-a}{\mathcal{L}(f)-a}=1-\frac{c}{a}$ for a constant $c$ with $\frac{c}{a} \neq 1$ and $N\left(r, 1-\frac{c}{a}\right)=S(r, f)$.

Theorem 2 Under the assumptions of Theorem 1, we further suppose that $f, a(z)(\not \equiv 0, \infty)$ and $a_{j}, j=1,2, \ldots, k$, are all entire, then we have either $f=\mathcal{L}(f)$, or $\frac{f-a}{\mathcal{L}(f)-a}=c$ for a constant $c \neq 0$ and $a(z)$ must also be a constant as well.

As suggested by one of the referees, the reader may also feel interested in a very recent paper by Al-Khaladi [18], where in fact some results of Lahiri and Sarkar [19] were generalized that in turn was deeply related to a paper of Yu [20]. One notices the paper [19] 
was not suitably cited in the key reference of [18] (not the paper [18] itself), despite the conclusions as well as the methods being very similar.

It is worth to mention that the machinery used in this paper is standard Nevanlinna's value distribution theory while the methods involved are combinations of those already applied in Al-Khaladi $[16,17]$ as well as in Han and Yi [8].

\section{Proofs of the main results}

This section is devoted to the detailed proofs of Theorems 1 and 2. We first define the following auxiliary function $\alpha$ as

$$
\alpha:=\frac{f-a}{\mathcal{L}-a} .
$$

Here and hereafter, we use $\mathcal{L}$ for $\mathcal{L}(f)$ for brevity. Our hypotheses imply $\alpha$ is such a meromorphic function that $T(r, \alpha)=O(T(r, f))$ yet $N(r, \alpha)=S(r, f)$.

Case I. $a$ is a finite, nonzero constant.

Rewrite (5) as $f-a=\alpha \mathcal{L}-a \alpha$ and differentiate it to yield

$$
f^{\prime}=(\alpha \mathcal{L})^{\prime}-a \alpha^{\prime}=\alpha\left(\beta f^{\prime}-\gamma\right),
$$

where we introduced two meromorphic functions $\beta:=\frac{(\alpha \mathcal{L})^{\prime}}{\alpha f^{\prime}}$ and $\gamma:=a \frac{\alpha^{\prime}}{\alpha}$.

Notice that $\alpha \not \equiv 0$. We get easily

$$
\left\{\begin{array}{l}
\frac{1}{\alpha}=\beta-\gamma \frac{1}{f^{\prime}}, \\
-\frac{\alpha^{\prime}}{\alpha^{2}}=\beta^{\prime}+\gamma \frac{f^{\prime \prime}}{\left(f^{\prime}\right)^{2}}-\gamma^{\prime} \frac{1}{f^{\prime}} .
\end{array}\right.
$$

Substitute $\frac{1}{\alpha}$ from the top line to the bottom line in (7) to derive

$$
\left(\gamma \frac{1}{f^{\prime}}-\beta\right) \frac{\alpha^{\prime}}{\alpha}=\beta^{\prime}+\gamma \frac{f^{\prime \prime}}{\left(f^{\prime}\right)^{2}}-\gamma^{\prime} \frac{1}{f^{\prime}} .
$$

That is, for the meromorphic function $\delta:=\gamma\left(\frac{\alpha^{\prime}}{\alpha}+\frac{\gamma^{\prime}}{\gamma}-\frac{f^{\prime \prime}}{f^{\prime}}\right)$, one has

$$
\delta \frac{1}{f^{\prime}}=\beta^{\prime}+\beta \frac{\alpha^{\prime}}{\alpha}
$$

Now, when $\gamma \equiv 0$, then we see $\alpha$ is a constant, so that $\frac{f-a}{\mathcal{L}(f)-a}=c$ for a constant $c=\alpha \neq 0$. As a consequence, we assume in the following that $\gamma \neq \equiv 0$.

Next, if $\delta \equiv 0$, then $\frac{\alpha^{\prime}}{\alpha}+\frac{\gamma^{\prime}}{\gamma}-\frac{f^{\prime \prime}}{f^{\prime}} \equiv 0$; using $\alpha \gamma=a \alpha^{\prime}$, one derives

$$
\alpha \gamma=a \alpha^{\prime}=c_{1} f^{\prime}
$$

Here, $c_{1} \neq 0$ is a constant. Hence, it follows that $N(r, f)=O(N(r, \alpha))=S(r, f)$, which further yields $N\left(r, \frac{1}{\alpha}\right) \leq k \bar{N}(r, f)+S(r, f)=S(r, f)$. On the other hand, combining (6) and (10) provides us with the following identity:

$$
\left(1+c_{1}\right) f^{\prime}=(\alpha \mathcal{L})^{\prime}
$$


If $c_{1}=-1$, then $\alpha \mathcal{L}=c_{2}$ for a constant $c_{2} \neq 0$; yet, since now $f^{\prime}=-a \alpha^{\prime}$, we have $\mathcal{L}=-a \mathcal{L}(\alpha)$ via an easy computation, so that $-a \alpha \mathcal{L}(\alpha)=c_{2}$. Rewrite it as

$$
\frac{c_{2}}{\alpha^{2}}=-a \frac{\mathcal{L}(\alpha)}{\alpha} .
$$

This then implies that $m\left(r, \frac{1}{\alpha}\right) \leq \frac{1}{2} m\left(r, \frac{\mathcal{L}(\alpha)}{\alpha}\right)+S(r, f)=S(r, f)$. Consequently, using the first main theorem, one has $T(r, \alpha)=S(r, f)$ and thus $T(r, f)=S(r, f)$ from (10). This is a contradiction. If $c_{1} \neq-1$, then from (11) one observes

$$
\frac{1+c_{1}}{\alpha}=\frac{(\alpha \mathcal{L})^{\prime}}{\alpha f^{\prime}}=\beta
$$

An easy calculation says $m\left(r, \frac{1}{\alpha}\right) \leq m(r, \beta)+S(r, f)=S(r, f)$, so that $T(r, \alpha)=S(r, f)$ and thus $T(r, f)=S(r, f)$ by $(10)$. This is a contradiction again.

All these foregoing discussions imply that $\delta \not \equiv 0$. So, from (9), we have

$$
\begin{aligned}
m\left(r, \frac{1}{f^{\prime}}\right) & \leq m\left(r, \frac{\alpha^{\prime}}{\alpha}\right)+m(r, \beta)+m\left(r, \frac{\beta^{\prime}}{\beta}\right)+m\left(r, \frac{1}{\delta}\right) \\
& =m\left(r, \frac{1}{\delta}\right)+S(r, f)=T(r, \delta)-N\left(r, \frac{1}{\delta}\right)+S(r, f) \\
& =m(r, \delta)+N(r, \delta)-N\left(r, \frac{1}{\delta}\right)+S(r, f) .
\end{aligned}
$$

Keeping in mind $m(r, \gamma)=S(r, f)$, we have $m(r, \delta)=S(r, f)$ and thus

$$
m\left(r, \frac{1}{f^{\prime}}\right)+N\left(r, \frac{1}{\delta}\right)=N(r, \delta)+S(r, f) .
$$

Reset $\delta=a\left(\frac{\alpha^{\prime}}{\alpha}\right)^{2}+a\left(\frac{\alpha^{\prime}}{\alpha}\right)^{\prime}-a \frac{\alpha^{\prime}}{\alpha} f_{f^{\prime}}$ and note that the zeros of $\alpha$ come from the poles of $f$. When $f^{\prime}\left(z_{0}\right)=0$ with multiplicity $p \geq k+1$, then $\alpha\left(z_{0}\right) \neq 0$ and $\mathcal{L}(f)\left(z_{0}\right)=(\mathcal{L}(f))^{\prime}\left(z_{0}\right)=0$ with multiplicities at least $p-k+1$ and $p-k$, respectively; using (6), one observes $\alpha^{\prime}\left(z_{0}\right)=0$ with multiplicity at least $p-k$ since $f^{\prime}-(\alpha \mathcal{L})^{\prime}=a \alpha^{\prime}$; thus, recalling $\gamma=a \frac{\alpha^{\prime}}{\alpha}$, we know $\gamma\left(z_{0}\right)=0$ with multiplicity at least $p-k$. Thereby, $\delta\left(z_{0}\right)=0$ with multiplicity at least $p-$ $k-1$. As a result, it follows that

$$
N_{(k+1}\left(r, \frac{1}{f^{\prime}}\right) \leq N\left(r, \frac{1}{\delta}\right)+(k+1) \bar{N}_{(k+1}\left(r, \frac{1}{f^{\prime}}\right)+S(r, f) .
$$

When $\delta\left(z_{1}\right)=\infty$, then either $\alpha\left(z_{1}\right)=0$ (from $f\left(z_{1}\right)=\infty$ in fact) or $f\left(z_{1}\right)=\infty$ with multiplicity 2 of $\delta\left(z_{1}\right)=\infty$, or $f^{\prime}\left(z_{1}\right)=0$ with multiplicity 1 of $\delta\left(z_{1}\right)=\infty$. In view of (15) for the case where $f^{\prime}\left(z_{1}\right)=0$, we can thus conclude that

$$
N(r, \delta) \leq 2 \bar{N}(r, f)+\bar{N}_{k)}\left(r, \frac{1}{f^{\prime}}\right)+S(r, f)
$$

Here, $N_{k)}\left(r, \frac{1}{f^{\prime}}\right), \bar{N}_{k)}\left(r, \frac{1}{f^{\prime}}\right)$, and $N_{(k+1}\left(r, \frac{1}{f^{\prime}}\right), \bar{N}_{(k+1}\left(r, \frac{1}{f^{\prime}}\right)$ are the counting functions of the zeros of $f^{\prime}$ with multiplicities $\leq k$ and $\geq k+1$, respectively. 
Altogether with (14), (15), and (16), we obtain

$$
\begin{aligned}
T\left(r, f^{\prime}\right) & =m\left(r, \frac{1}{f^{\prime}}\right)+N_{k)}\left(r, \frac{1}{f^{\prime}}\right)+N_{(k+1}\left(r, \frac{1}{f^{\prime}}\right) \\
& =N(r, \delta)+N_{k)}\left(r, \frac{1}{f^{\prime}}\right)+(k+1) \bar{N}_{(k+1}\left(r, \frac{1}{f^{\prime}}\right)+S(r, f) \\
& \leq 2 \bar{N}(r, f)+\bar{N}_{k)}\left(r, \frac{1}{f^{\prime}}\right)+N_{k)}\left(r, \frac{1}{f^{\prime}}\right)+(k+1) \bar{N}_{(k+1}\left(r, \frac{1}{f^{\prime}}\right)+S(r, f),
\end{aligned}
$$

which further implies that, as $k \geq 1$ and $N_{k)}\left(r, \frac{1}{f^{\prime}}\right) \leq k \bar{N}_{k)}\left(r, \frac{1}{f^{\prime}}\right)+S(r, f)$,

$$
T\left(r, f^{\prime}\right) \leq(k+1)\left\{\bar{N}(r, f)+\bar{N}\left(r, \frac{1}{f^{\prime}}\right)\right\}+S(r, f) .
$$

This obviously contradicts our assumption. As a result, when $a$ is a finite, nonzero constant, it follows that $\frac{f-a}{\mathcal{L}(f)-a}=c$ with a constant $c \neq 0$.

Case II. $a$ is a nonconstant, small function.

Like before, rewrite (5) as $f-a=\alpha \mathcal{L}-a \alpha$ and differentiate it to yield

$$
f^{\prime}-a^{\prime}=(\alpha \mathcal{L})^{\prime}-(\alpha a)^{\prime}
$$

When $\left(f^{\prime}-a^{\prime}\right)\left(z_{2}\right)=0$, then we have $\left\{(\alpha \mathcal{L})^{\prime}-(\alpha a)^{\prime}\right\}\left(z_{2}\right)=0$. Recall that the zeros of $\alpha$ come from the poles of $f$. As a consequence, one deduces that

$$
H\left(z_{2}\right):=\left\{\frac{(\alpha \mathcal{L})^{\prime}}{\alpha f^{\prime}}-\frac{(a \alpha)^{\prime}}{\alpha a^{\prime}}\right\}\left(z_{2}\right)=0 .
$$

If $H \not \equiv 0$, then we have, noticing that $m(r, H)=S(r, f)$,

$$
\begin{aligned}
\bar{N}\left(r, \frac{1}{f^{\prime}-a^{\prime}}\right) & \leq N\left(r, \frac{1}{H}\right)+S(r, f) \\
& =T(r, H)+S(r, f)=N(r, H)+S(r, f) .
\end{aligned}
$$

Note when $H\left(z_{3}\right)=\infty$, then we have either $\alpha\left(z_{3}\right)=0$ (from $f\left(z_{3}\right)=\infty$ actually) or $f\left(z_{3}\right)=\infty$ - with multiplicity $k$ of $H\left(z_{3}\right)=\infty$, or $f^{\prime}\left(z_{3}\right)=0$ - with multiplicity $k-1$ of $H\left(z_{3}\right)=\infty$. Thus, we can derive that, similar to (16),

$$
N(r, H) \leq k\left\{\bar{N}(r, f)+\bar{N}\left(r, \frac{1}{f^{\prime}}\right)\right\}+S(r, f) .
$$

Apply the second main theorem for three small functions to $f^{\prime}$ to yield

$$
T\left(r, f^{\prime}\right) \leq \bar{N}\left(r, \frac{1}{f^{\prime}}\right)+\bar{N}\left(r, f^{\prime}\right)+\bar{N}\left(r, \frac{1}{f^{\prime}-a^{\prime}}\right)+S(r, f),
$$

which together with (20) yields (17) again. This gives a contradiction.

Thus, $H \equiv 0$. That is, $(\alpha \mathcal{L})^{\prime} \equiv \frac{f^{\prime}}{a^{\prime}}(a \alpha)^{\prime}$. Using (18), one derives

$$
a^{\prime}\left(f^{\prime}-a^{\prime}\right) \equiv(a \alpha)^{\prime}\left(f^{\prime}-a^{\prime}\right),
$$


so that $a^{\prime} \equiv(a \alpha)^{\prime}$; in other words, $\alpha=1-\frac{c}{a}$ for a constant $c$ with $\frac{c}{a} \neq 1$. We thus conclude that $\frac{f-a}{\mathcal{L}(f)-a}=1-\frac{c}{a}$ and $N\left(r, 1-\frac{c}{a}\right)=N(r, \alpha)=S(r, f)$.

Finally, let us prove the associated Theorem 2 . When $f, a$, and $a_{j}(j=1,2, \ldots, k)$ are all entire functions, we have $\alpha(z)=e^{\omega(z)}$ for some entire function $\omega(z)$. If $a$ is constant, then we have the same result as in Case I: $\frac{f-a}{\mathcal{L}(f)-a}=c$ with a constant $c \neq 0$; if $a$ is nonconstant, then $\frac{c}{a}=1-e^{\omega(z)}$ admits many zeros, so that $a$ cannot be entire unless $c=0$, which provides us with the case $f=\mathcal{L}(f)$.

\section{Competing interests}

The authors declare that they have no competing interests.

\section{Authors' contributions}

All authors contributed equally to the writing of this paper. All authors read and approved the final manuscript.

\section{Author details}

'Department of Mathematics, Jining University, Qufu, Shandong 273155, P.R. China. ²Department of Mathematics, University of Houston, Houston, Texas 77204, USA.

\section{Acknowledgements}

Both authors are greatly indebted to the anonymous referees.

Received: 29 March 2014 Accepted: 1 October 2014 Published: 13 October 2014

\section{References}

1. Hayman, WK: Meromorphic Functions. Clarendon Press, Oxford (1964)

2. Yang, L: Value Distribution Theory. Springer, Berlin (1993)

3. Yi, HX, Yang, CC: Uniqueness Theory of Meromorphic Functions. Kluwer Academic, Dordrecht (2003)

4. Rubel, LA, Yang, CC: Values Shared by an Entire Function and Its Derivative. Lecture Notes in Math., vol. 599. Springer, New York (1977)

5. Gundersen, GG: Meromorphic functions that share two finite values with their derivative. Pac. J. Math. 105, 299-309 (1983)

6. Mues, E, Steinmetz, N: Meromorphe Funktionen, die mit ihrer Ableitung zwei Werte teilen. Results Math. 6, 48-55 (1983)

7. Han, Q, Hu, PC: Unicity of meromorphic functions related to their derivatives. Bull. Belg. Math. Soc. Simon Stevin 14, 905-918 (2007)

8. Han, Q, Yi, HX: On the uniqueness problems of entire functions and their linear differential polynomials. Kodai Math. J. 30, 61-73 (2007)

9. Han, Q, Liu, J: Unicity of entire functions and a related problem. Commun. Math. Anal. 9, 42-50 (2010)

10. Li, P, Yang, CC: Value sharing of an entire function and its derivatives. J. Math. Soc. Jpn. 51, 781-799 (1999)

11. Li, P, Yang, CC: When an entire function and its linear differential polynomial share two values. III. J. Math. 44, 349-362 (2000)

12. Li, $\mathrm{P}$, Yang, CC: Uniqueness theorems on entire functions and their derivatives. J. Math. Anal. Appl. 253, 50-57 (2001)

13. Li, P: Unicity of meromorphic functions and their derivatives. J. Math. Anal. Appl. 285, 651-665 (2003)

14. Brück, R: On entire functions which share one value CM with their first derivative. Results Math. 30, $21-24$ (1996)

15. Zhang, QC: The uniqueness of meromorphic functions with their derivatives. Kodai Math. J. 21, 179-184 (1998)

16. Al-Khaladi, AHH: On entire functions which share one small function CM with their kth derivative. Results Math. 47, 1-5 (2005)

17. Al-Khaladi, $\mathrm{AHH}$ : On meromorphic functions that share one small function with their kth derivative. Results Math. $\mathbf{5 7}$, 313-318 (2010)

18. Al-Khaladi, AHH: Meromorphic functions that share a small function with one of its linear differential polynomials. Afr. Math. 25, 567-575 (2014)

19. Lahiri, I, Sarkar, A: Uniqueness of a meromorphic function and its derivative. JIPAM. J. Inequal. Pure Appl. Math. 5(1), Article 20 (2004)

20. Yu, KW: On entire and meromorphic functions that share small functions with their derivatives. JIPAM. J. Inequal. Pure Appl. Math. 4(1), Article 21 (2003) 